\title{
Discontinuation of antiepileptic drugs in adults with epilepsy
}

\section{SUMMARY}

Patients with epilepsy who have been free of seizures for at least two years may be able to stop their antiepileptic drugs. Discontinuation may be considered after an individualised harm-benefit assessment and consultation with a neurologist is recommended.

It is paramount to discuss with patients whether the risk of seizure recurrence is worth the benefit of stopping the antiepileptic drug.

The risk of seizure recurrence after antiepileptic drugs are discontinued depends on the epilepsy syndrome and a number of other risk factors. Approximately 30-50\% of patients will relapse.

If seizures recur, the majority of patients regain seizure control when treatment is resumed. However up to $20 \%$ do not achieve immediate remission.

\section{Introduction}

Antiepileptic drugs are effective in stopping seizures in approximately two-thirds of patients with newly diagnosed epilepsy. 'However, it is impossible to know in patients who are seizure-free for a long time whether the absence of seizures is due to seizure suppression by their treatment, or due to remission of the epilepsy.

A decision to continue or to stop antiepileptic drug treatment requires an individualised harm-benefit assessment. The main concern is the recurrence of seizures after treatment stops. A number of factors need to be considered when assessing the risk of recurrence. It is important to include patients in the discussion of whether this risk is worth the benefit of stopping treatment.

\section{Reasons to consider discontinuation}

Up to $88 \%$ of patients experience adverse effects from antiepileptic drugs. These include dizziness, sedation, cognitive and neuropsychiatric symptoms, which can negatively affect quality of life. ${ }^{2}$ There are also concerns regarding bone health and an increased risk of fractures as a long-term complication with some antiepileptic drugs. ${ }^{3}$

Women of childbearing age often worry about the potential teratogenicity of antiepileptic drugs. This may be a motivation to attempt to reduce their antiepileptic drugs, ideally long before actually planning a pregnancy. While it is important to limit exposure to teratogenic antiepileptic drugs during pregnancy, abrupt cessation should be avoided. Some antiepileptic drugs have a known (often dosedependent) risk of teratogenicity. Valproate has the highest risk of major congenital malformations. Other drugs are considered safer with lamotrigine and levetiracetam having the lowest risk. ${ }^{4}$ For many newer antiepileptic drugs the risk of harm is still unknown.

Antiepileptic drugs, such as carbamazepine and phenytoin, affect important hepatic enzyme systems such as cytochrome P450. They can have significant pharmacological interactions such as reducing the efficacy of oral contraceptive pills, oral anticoagulants (warfarin and direct oral anticoagulants) and directacting antiviral drugs for chronic hepatitis $\mathrm{C}$. Patients with chronic hepatitis $C$ are usually required to either taper their therapy or switch to an alternative antiepileptic drug before starting antiviral treatment.

Other reasons for discontinuation may include the cost of treatment. There is also the wish to feel 'cured' and to avoid the inconvenience and stigma of taking drugs daily.

The discussion about antiepileptic drug discontinuation should prompt review of the original diagnosis and supporting evidence. Patients with an equivocal history of seizures or patients who never fulfilled the diagnostic criteria for epilepsy (e.g. acute symptomatic seizures or prophylactic use of an antiepileptic drug) should be evaluated again to see if there is any indication for continuing treatment.

It is important to explore the patient's concerns and motivation for antiepileptic drug withdrawal. There may be alternatives for the patient to consider such as dose reduction or change of antiepileptic drug to address adverse effects, pharmacological interactions or teratogenicity. Some patients may only need clarification and reassurance regarding the safety profile of their antiepileptic drug.

\section{Hanka Laue-Gizzi iD \\ Consultant neurologist and Epileptologist, Comprehensive Epilepsy Service, Prince of Wales Hospital \\ Conjoint lecturer, University of New South Wales \\ Sydney}

\section{Keywords}

anticonvulsants, epilepsy

Aust Prescr 2021:44:53-6 https://doi.org/10.18773/ austprescr.2021.005 


\section{Risk of seizure recurrence}

The main risk associated with discontinuing antiepileptic drug therapy is seizure recurrence. This occurs in $26-63 \%$ of cases depending on the patient population. ${ }^{5-7}$ A meta-analysis of 10 studies including 1769 patients with varying characteristics found a seizure recurrence rate of $46 \%$ after antiepileptic drugs were stopped. ${ }^{5}$ The rate of seizure recurrence within the same time period is about twice the rate reported with continued treatment. ${ }^{6,8}$ The risk is highest within the first 6-12 months after discontinuation, but remains substantially increased for many years. ${ }^{5,6}$

Seizure recurrence can have devastating physical, psychological and social consequences. These may include injury, loss of self-esteem, stigma around seizures, unemployment and the inability to drive.

Some patients are willing to stop antiepileptic drugs even when the risk of relapse is substantial, whereas others fear the return of seizures and decide to continue their antiepileptic drugs. In one study, more than half of the patients preferred to continue their antiepileptic drug after two years of seizure freedom. They felt well-adjusted to their treatment and were concerned about possible seizure recurrence after withdrawal and the subsequent loss of their driving licence or even their jobs. ${ }^{9}$

\section{Factors associated with seizure recurrence}

A large meta-analysis identified independent predictors of seizure recurrence after treatment stops (see Boxes 1 and 2). ${ }^{5}$ The authors of the analysis created an easy-to-use web-based epilepsy prediction tool to assist clinicians when counselling patients. This tool is particularly useful in patients with some predictors in favour and others against stopping treatment. It calculates an individualised risk of seizures in the next two and five years after antiepileptic drug withdrawal, and the chance to be seizure-free

\section{Box 1 Factors associated with an increased risk of seizure recurrence ${ }^{5}$}

Long duration of epilepsy before remission

Short seizure-free interval before antiepileptic drug withdrawal

Older age at onset of epilepsy (in patients $>25$ years)

History of febrile seizures

More than 10 seizures before remission

Absence of a self-limiting epilepsy syndrome

e.g. absence or rolandic epilepsy

Developmental delay

Epileptiform abnormality on EEG before withdrawal after 10 years. The calculator should not be used as a substitute for an individualised discussion of the full range of harms and benefits, but it helps substantially to guide tailored choices by the doctor and patient.

The type of epilepsy should always be included in the decision-making process before treatment is discontinued. The risk of seizure recurrence even after many years of being seizure-free is particularly high for patients with juvenile myoclonic epilepsy or focal epilepsy with a structural aetiology, which are the most common epilepsies in adults. $5,8,10$

\section{Important considerations for counselling}

Patients need to understand the potential problems that can occur after stopping treatment, particularly the consequences of relapse.

\section{Regaining seizure control after relapse}

If patients have seizures after stopping treatment, they have a good chance of becoming seizure-free again by resuming their drugs. However, up to $20 \%$ of patients do not achieve immediate remission and, for some patients, it may take several years to become seizure-free again."

\section{Driving}

The implications for driving when discontinuing treatment are important to discuss with the patient. This may be the sole reason a patient decides against stopping antiepileptic drugs.

In Australia patients must stop driving while being weaned off antiepileptic drugs and for an additional three months after the last dose, in accordance with the Assessing Fitness to Drive Framework (private vehicle driver standard).12 It is reasonable to extend this period in patients with a previously low seizure frequency to confirm that their freedom from seizures is sustained. If there is seizure recurrence, patients may resume driving if the previously effective treatment is resumed and there have been no seizures for four weeks.

\section{Box 2 Factors associated with long-term seizure freedom (at 10 years after antiepileptic drug withdrawal) ${ }^{5}$}

Short duration of epilepsy before remission

Long seizure-free interval (years) before antiepileptic drug withdrawal

One or low number of antiepileptic drugs before withdrawal

Low number of seizures before remission

No history of focal seizures

No epileptiform abnormality on EEG before withdrawal 


\section{Drugs with other indications}

Several antiepileptic drugs have additional beneficial effects, for example mood stabilisation in bipolar disorder (valproate, carbamazepine and lamotrigine) and migraine prophylaxis (topiramate, valproate). Monitoring for recurrence of these symptoms after the antiepileptic drug is withdrawn is recommended for patients who have these comorbidities.

\section{Psychosocial impact}

The antiepileptic drug weaning period may be associated with significant anxiety. This can be regarding seizure recurrence, restricted social activities and a possible impact on employment and driving.

\section{Drug withdrawal after surgery for epilepsy}

Resective epilepsy surgery aims to remove the epileptogenic zone and offers the chance of a cure. The risk of seizure recurrence after discontinuing antiepileptic drugs is one in three for patients who are seizure-free following surgery. ${ }^{13}$ There are no guidelines for postoperative antiepileptic drug withdrawal in seizure-free patients. Practices vary widely across specialist centres, however, one year is a common time frame. The decision if and when to stop antiepileptic drugs must be individualised. It depends on the type of epilepsy surgery, aetiology of the epilepsy, the completeness of the resection and the patient's attitude to discontinuation. This is usually discussed as part of the postoperative care at the epilepsy centre.

\section{When to stop antiepileptic drugs}

Drug discontinuation may be considered after a minimum of two years without a seizure. The risk of recurrence decreases with every additional year of seizure freedom. ${ }^{5,10}$ However, the two-year threshold is an artificial construct and should now be replaced by an individualised approach and a thorough examination of all the risks and benefits for each patient. ${ }^{5}$

In patients with an increased risk of seizure recurrence, it is advisable to wait longer than two years before considering antiepileptic drug discontinuation. The same is true for patients who had a low frequency of seizures (e.g. less than once a year) before remission.

Patients with a significant risk of seizure recurrence should not be encouraged or even advised to discontinue antiepileptic drugs even after a long period of seizure freedom. They include patients with juvenile myoclonic epilepsy, who only have a small chance of successful antiepileptic drug withdrawal. ${ }^{14}$

\section{How to stop antiepileptic drugs}

Most guidelines encourage slow discontinuation of antiepileptic drugs. However, the duration of the tapering period varies greatly and depends on multiple factors such as the number of antiepileptic drugs, the starting dose, previous seizure frequency, seizure type and associated risk of injury, risk of withdrawal seizures with some antiepileptic drugs and the non-driving period. Patients on multiple antiepileptic drugs need to withdraw them sequentially. The tapering schedule should ideally be provided by a neurologist after taking all relevant factors into account and discussing them with the patient. The taper duration will also be influenced by the patient's needs and preferences. ${ }^{10}$ A slow taper (e.g. over months) allows observation and helps to document the minimally effective doses in case the seizures recur. However, a slow taper prolongs the non-driving period

The taper rate for benzodiazepines (especially clonazepam) and barbiturates should be particularly slow. This reduces the risk of withdrawal symptoms and withdrawal seizures.

\section{Management of seizure recurrence}

If seizures recur, the previously effective drug should be restarted unless it was discontinued because of unacceptable side effects, in which case an alternative could be tried. Similarly, a woman of childbearing age whose seizures recur after stopping a teratogenic antiepileptic drug could be started on a drug with a lower teratogenic risk.

\section{Conclusion}

The decision to continue or to stop antiepileptic drugs in seizure-free adults requires an individualised harm-benefit assessment. The risk of seizure recurrence depends on a number of factors and there are tools available to assess this in individual patients. Social aspects such as driving and employment, as well as emotional and personal factors, must be carefully considered along with adverse effects and drug interactions. The doctor's role is to provide detailed information to help the patient make an informed decision. $\varangle$

Conflicts of interest: none declared
SELF-TEST QUESTIONS

True or false?

1. Patients with juvenile myoclonic epilepsy can successfully stop antiepileptic drugs when they reach adulthood.

2. When discontinuing treatment for patients taking multiple antiepileptic drugs, the drugs should be withdrawn sequentially.

Answers on page 7 


\section{REFERENCES}

1. Kwan P, Brodie MJ. Early identification of refractory epilepsy. N Engl J Med 2000;342:314-9. https://doi.org/10.1056/ NEJM200002033420503

2. Baker GA, Jacoby A, Buck D, Stalgis C, Monnet D. Quality of life of people with epilepsy: a European study. Epilepsia 1997;38:353-62. https://doi.org/10.1111/j.1528-1157.1997.tb01128.x

3. Carbone LD, Johnson KC, Robbins J, Larson JC, Curb JD, Watson K, et al. Antiepileptic drug use, falls, fractures, and BMD in postmenopausal women: findings from the Women's Health Initiative (WHI). J Bone Miner Res 2010;25:873-81. https://doi.org/10.1359/jbmr.091027

4. Tomson T, Battino D, Bromley R, Kochen S, Meador K, Pennell $P$, et al. Management of epilepsy in pregnancy: a report from the International League Against Epilepsy Task Force on Women and Pregnancy. Epileptic Disord 2019;21:497-517. https://doi.org/10.1684/epd.2019.1105

5. Lamberink HJ, Otte WM, Geerts AT, Pavlovic M, Ramos-Lizana J, Marson AG, et al. Individualised prediction model of seizure recurrence and long-term outcomes after withdrawal of antiepileptic drugs in seizure-free patients: a systematic review and individual participant data metaanalysis. Lancet Neurol 2017;16:523-31. https://doi.org/ 10.1016/S1474-4422(17)30114-X

6. Medical Research Council Antiepileptic Drug Withdrawal Study Group. Randomised study of antiepileptic drug withdrawal in patients in remission. Lancet 1991;337:1175-80.

7. Berg AT, Shinnar S. Relapse following discontinuation of antiepileptic drugs: a meta-analysis. Neurology 1994:44:601-8. https://doi.org/10.1212/WNL.44.4.601

8. Schmidt D, Sillanpää M. Stopping epilepsy treatment in seizure remission: Good or bad or both? Seizure 2017:44:157-61. https://doi.org/10.1016/j.seizure.2016.09.003
9. Cvetkovska E, Babunovska M, Kuzmanovski I, Boskovski B, Sazdova-Burneska S, Aleksovski V, et al. Patients' attitude toward AED withdrawal: a survey among individuals who had been seizure-free for over 2 years. Epilepsy Behav. Epub 2020 Jan 11. https://doi.org/10.1016/j.yebeh.2019.106881

10. Beghi E, Giussani G, Grosso S, ludice A, La Neve A, Pisani F, et al. Withdrawal of antiepileptic drugs: guidelines of the Italian League Against Epilepsy. Epilepsia 2013;54(Suppl 7):2-12. https://doi.org/10.1111/epi.12305

11. Schmidt D, Löscher W. Uncontrolled epilepsy following discontinuation of antiepileptic drugs in seizurefree patients: a review of current clinical experience. Acta Neurol Scand 2005;111:291-300. https://doi.org/10.1111/ j.1600-0404.2005.00408.x

12. Assessing fitness to drive for commercial and private vehicle drivers. 2016 Medical standards for licensing and clinical management guidelines. Sydney: Ausroads. p. 93. https://austroads.com.au/publications/assessing-fitness-todrive/ap-g56 [cited 2021 Mar 1]

13. Schmidt D, Baumgartner C, Löscher W. Seizure recurrence after planned discontinuation of antiepileptic drugs in seizure-free patients after epilepsy surgery: a review of current clinical experience. Epilepsia 2004:45:179-86. https://doi.org/10.1111/j.0013-9580.2004.37803.x

14. Höfler J, Unterberger I, Dobesberger J, Kuchukhidze G, Walser G, Trinka E. Seizure outcome in 175 patients with juvenile myoclonic epilepsy--a long-term observational study. Epilepsy Res 2014;108:1817-24. https://doi.org/10.1016/ j.eplepsyres.2014.09.008 